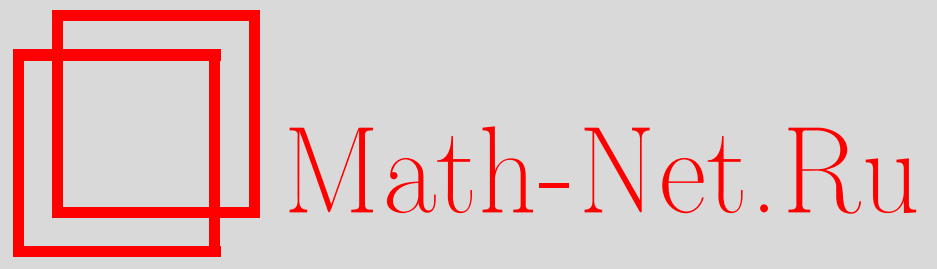

G. Peskir, Optimal Stopping Games and Nash Equilibrium, Теория вероятн. и ее примен., 2008, том 53, выпуск 3, 623-638

DOI: https://doi.org/10.4213/tvp2457

Использование Общероссийского математического портала Math-Net.Ru подразумевает, что вы прочитали и согласны с пользовательским соглашением

http://www . mathnet.ru/rus/agreement

Параметры загрузки:

IP : 52.23 .180 .231

26 апреля 2023 г., $07: 11: 01$ 
(C) $2008 \Gamma$.

PESKIR G.*

\section{OPTIMAL STOPPING GAMES AND NASH EQUILIBRIUM}

Показывается, что функция цены в игровой задаче об оптимальной остановке для непрерывного справа сильно марковского процесса может быть найдена из равенства наименьшей супергармонической и наибольшей субгармонической функций, лежащих между функциями выигрыша (семигармоническая характеризация) тогда u только тогда, когда имеет место равновесие по Нэшу (т.е. когда существует седловая пара оптимальных моментов остановки). В применении к задачам оптимальной остановки показано, что нахождение функции цены сводится к классической характеризации этой функции в терминах супергармонических и субгармонических функций. Отмеченная эквивалентность показывает, что нахождение функции цены «протягиванием веревки» между «двумя препятствиями» равнозначно доказательству равновесия по Нэшу. В ходе доказательства устанавливаются различные свойства функции цены и оптимальных моментов остановки.

Ключевые слова и фразы: игровая задача об оптимальной остановке, равновесие по Нэшу, семигармоническая характеризация функции цены, задача со свободными границами, принцип гладкого склеивания, принцип непрерывного склеивания, оптимальная остановка, марковский процесс, семимартингал.

1. Introduction. Consider the optimal stopping game, where the supplayer chooses a stopping time $\tau$ to maximize, and the inf-player chooses a stopping time $\sigma$ to minimize, the expected payoff

$$
\begin{aligned}
\mathrm{M}_{x}(\tau, \sigma)=\mathrm{E}_{x}[ & G_{1}\left(X_{\tau}\right) I(\tau<\sigma) \\
& \left.\quad+G_{2}\left(X_{\sigma}\right) I(\sigma<\tau)+G_{3}\left(X_{\tau}\right) I(\tau=\sigma)\right],
\end{aligned}
$$

where $X=\left(X_{t}\right)_{t \geqslant 0}$ is a strong Markov process with $X_{0}=x$ un$\operatorname{der} \mathbf{P}_{x}$, and $G_{1}, G_{2}$, and $G_{3}$ are (finely) continuous functions satisfying $G_{1} \leqslant G_{3} \leqslant G_{2}$. Define the upper value and the lower value of the game by

$$
V^{*}(x)=\inf _{\sigma} \sup _{\tau} \mathrm{M}_{x}(\tau, \sigma) \quad \text { and } \quad V_{*}(x)=\sup _{\tau} \inf _{\sigma} \mathrm{M}_{x}(\tau, \sigma),
$$

* School of Mathematics, The University of Manchester, Oxford Road, Manchester M13 9PL, United Kingdom; e-mail: goran@maths.man.ac.uk 
where the horizon (the upper bound for $\tau$ and $\sigma$ above) may be either finite or infinite (for further details of these hypotheses see Section 2 below). Note that $V_{*}(x) \leqslant V^{*}(x)$ for all $x$ and recall that in this context one distinguishes: (i) the Stackelberg equilibrium, meaning that

$$
V^{*}(x)=V_{*}(x)
$$

for all $x$ (in this case $V:=V^{*}=V_{*}$ unambiguously defines the value of the game); and (ii) the Nash equilibrium, meaning that there exist stopping times $\tau_{*}$ and $\sigma_{*}$ such that

$$
\mathrm{M}_{x}\left(\tau, \sigma_{*}\right) \leqslant \mathrm{M}_{x}\left(\tau_{*}, \sigma_{*}\right) \leqslant \mathrm{M}_{x}\left(\tau_{*}, \sigma\right)
$$

for all stopping times $\tau$ and $\sigma$, and for all $x$ (in other words, $\left(\tau_{*}, \sigma_{*}\right)$ is a saddle point). It is easily seen that the Nash equilibrium implies the Stackelberg equilibrium with $V(x)=\mathrm{M}_{x}\left(\tau_{*}, \sigma_{*}\right)$ for all $x$.

A variant of the problem above was first studied by Dynkin [10] using martingale methods similar to those of Snell [29]. Specific examples of the same problem were studied in [15] and [18] using Markovian methods (see also [19] for martingale methods). In parallel to that Bensoussan and Friedman (cf. [16], [4], [5]) developed an analytic approach (for diffusions) based on variational inequalities. Martingale methods were further advanced in [25] (see also [31]), and Markovian setting was studied in [14] (via Wald-Bellman equations) and [30] (via penalty equations). More recent papers on optimal stopping games include [20], [23], [1], [17], [11], [13], [21], [22], [2], and [3]. These papers study specific problems and often lead to explicit solutions. For optimal stopping games with randomized stopping times see [24] and the references therein. For connections with singular stochastic control (forward/backward SDE) see [7] and the references therein. For non zero-sum optimal stopping games see [26] and the references therein (the optimal stopping game (1.2) is a zero-sum game, since the payoff (1.1) may be thought of as the payment of the inf-player to the sup-player if both players are viewed to be rational).

It was recently proved in [12] that if $X$ is right-continuous, then the Stackelberg equilibrium (1.3) holds with $V:=V^{*}=V_{*}$ defining a measurable function, and if $X$ is right-continuous and left-continuous over stopping times, then the Nash equilibrium (1.4) holds with

$$
\tau_{*}=\inf \left\{t \geqslant 0: X_{t} \in D_{1}\right\} \quad \text { and } \sigma_{*}=\inf \left\{t \geqslant 0: X_{t} \in D_{2}\right\},
$$

where $D_{1}=\left\{V=G_{1}\right\}$ and $D_{2}=\left\{V=G_{2}\right\}$. The two sufficient conditions are known to be most general in optimal stopping theory (see, e.g., [27] and [28]). Moreover, if $X$ is only right-continuous and not left-continuous over stopping times, then the Nash equilibrium can break down while the Stackelberg equilibrium still holds (cf. [12, Example 3.1]). 
On the other hand, a fundamental result in optimal stopping theory states that the value function $\hat{V}$ of the optimal stopping problem

$$
\hat{V}(x)=\sup _{\tau} \mathbf{E}_{x} G_{1}\left(X_{\tau}\right)
$$

is the smallest superharmonic function that lies above the gain function $G_{1}$, and likewise the value function $\breve{V}$ of the optimal stopping problem

$$
\check{V}(x)=\inf _{\tau} \mathbf{E}_{x} G_{2}\left(X_{\tau}\right)
$$

is the largest subharmonic function that lies below the gain function $G_{2}$. This result dates back to Dynkin [8] and was derived in parallel to the general supermartingale or submartingale characterization due to Snell [29] (for more details see, e.g., [27] and [28]). The characterization leads to the familiar picture, where $\hat{V}$ is identified with a rope put above the obstacle $G_{1}$ having both ends pulled to the ground (see Fig. 1), and likewise $\breve{V}$ is identified with a rope put below the obstacle $G_{2}$ having both ends pulled to the sky (both pictures refer to the case when $X$ is a standard Brownian motion killed at the end points of the interval).

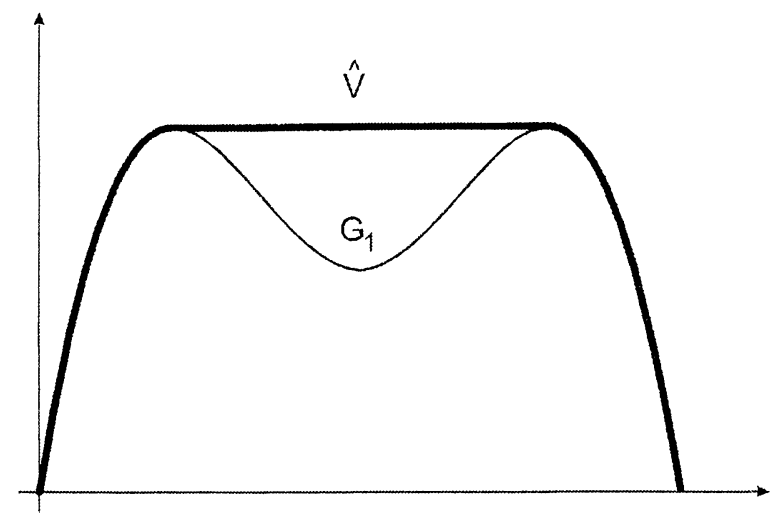

Fig. 1. An obstacle $G_{1}$ and the rope $\hat{V}$ depicting the superharmonic characterization of the value function in an optimal stopping problem

If we formally set $G_{2} \equiv+\infty$ in (1.1), then the optimal stopping game (1.2) reduces to the optimal stopping problem (1.6) and hence the value function $V=\hat{V}$ admits the superharmonic characterization. Likewise, if we formally set $G_{1} \equiv-\infty$ in (1.1), then the optimal stopping game (1.2) reduces to the optimal stopping problem (1.7) and hence the value function $V=\breve{V}$ admits the subharmonic characterization. This raises the question whether there is a semiharmonic characterization in the general case (when $G_{1}$ and $G_{2}$ are finite valued). A variant of this question was considered earlier under conditions which imply the Nash equilibrium at the first 
entry times (see [30]), and a one-sided version of the same question (where $V$ equals $\hat{V}$ ) was studied more recently when $X$ is a one-dimensional diffusion (see [11] and [13]).

The main purpose of the present paper is to address the question of the semiharmonic characterization in the general case, where $X$ is assumed to be a right-continuous strong Markov process (and no Nash equilibrium is assumed to be attained at the first entry times a priori). Our main result (Theorem 2.1) can be less formally stated as follows. Letting $\hat{V}$ denote the smallest superharmonic function lying between $G_{1}$ and $G_{2}$, and letting $\check{V}$ denote the largest subharmonic function lying between $G_{1}$ and $G_{2}$, we have $\hat{V}=\check{V}$ if and only if the Nash equilibrium (1.4) holds. Either (and thus both) of these facts will hold when $X$ is left-continuous over stopping times (additionally to right-continuity). The equivalence itself shows that finding the value function $V$ is the same as «pulling a rope» between «two obstacles» (see Fig. 2) which in turn is equivalent to establishing a Nash equilibrium. Further properties of the value function and the optimal stopping times are exhibited in the proof.

2. Semiharmonic characterization. 1. Throughout we will consider a strong Markov process $X=\left(X_{t}\right)_{t \geqslant 0}$ defined on a filtered probability space $\left(\Omega, \mathscr{F},\left(\mathscr{F}_{t}\right)_{t \geqslant 0}, \mathbf{P}_{x}\right)$ and taking values in a measurable space $(E, \mathscr{B})$, where $E$ is a locally compact Hausdorff space with a countable base, and $\mathscr{B}$ is the Borel $\sigma$-algebra on $E$. It will be assumed that the process $X$ starts at $x$ under $\mathbf{P}_{x}$ for $x \in E$ and that the sample paths of $X$ are right-continuous. Recall also that $X$ is said to be left-continuous over stopping times (quasileft-continuous) if $X_{\tau_{n}} \rightarrow X_{\tau} \mathbf{P}_{x}$-a.s. whenever $\tau_{n}$ and $\tau$ are stopping times such that $\tau_{n} \uparrow \tau$ as $n \rightarrow \infty$. (Stopping times are always referred with respect to the filtration $\left(\mathscr{F}_{t}\right)_{t \geqslant 0}$ given above.) It will also be assumed that the filtration $\left(\mathscr{F}_{t}\right)_{t \geqslant 0}$ is right-continuous (implying that the first entry times to open and closed sets are stopping times) and that $\mathscr{F}_{0}$ contains all $\mathbf{P}_{x}$-null sets from $\mathscr{F}_{\infty}^{X}=\sigma\left(X_{t}: t \geqslant 0\right)$ (implying also that the first entry times to Borel sets are stopping times). The main example we have in mind is when $\mathscr{F}_{t}=\sigma\left(\mathscr{F}_{t}^{X} \cup \mathscr{N}\right)$, where $\mathscr{F}_{t}^{X}=\sigma\left(X_{s}: 0 \leqslant s \leqslant t\right)$ and $\mathscr{N}=\left\{A \subseteq \Omega: \exists B \in \mathscr{F}_{\infty}^{X}, A \subseteq B, \mathbf{P}_{x}(B)=0\right\}$ for $t \geqslant 0$ with $\mathscr{F}=\mathscr{F}_{\infty}$. In addition, it is assumed that the mapping $x \mapsto \mathbf{P}_{x}(F)$ is (universally) measurable for each $F \in \mathscr{F}$. It follows that the mapping $x \mapsto \mathbf{E}_{x}(Z)$ is (universally) measurable for each (integrable) random variable $Z$. Finally, without loss of generality we will assume that $\Omega$ equals the canonical space $E^{[0, \infty)}$ with $X_{t}(\omega)=\omega(t)$ for $\omega \in \Omega$ and $t \geqslant 0$, so that the shift operator $\theta_{t}: \Omega \rightarrow \Omega$ is well defined by $\theta_{t}(\omega)(s)=\omega(t+s)$ for $\omega \in \Omega$ and $t, s \geqslant 0$.

The latter hypothesis enables one to use the following fact (which can be derived using Galmarino's test): If $\sigma \leqslant \tau$ are stopping times (not necessarily the first entry times), then there exists a function $\tau_{\sigma}: \Omega \times \Omega \rightarrow[0, \infty]$ 
such that

$$
\begin{gathered}
\tau_{\sigma} \quad \text { is } \mathscr{F}_{\sigma} \otimes \mathscr{F}_{\infty} \text {-measurable, } \\
\vartheta \longmapsto \tau_{\sigma}(\omega, \vartheta) \text { is a stopping time, } \\
\tau(\omega)=\sigma(\omega)+\tau_{\sigma}\left(\omega, \theta_{\sigma}(\omega)\right)
\end{gathered}
$$

for all $\omega \in \Omega$. If $\tau$ is the first entry time of $X$ into a set, then $\tau=\sigma+\tau \circ \theta_{\sigma}$ and $\tau_{\sigma}$ above may be identified with $\tau$ (in the sense that $\tau_{\sigma}(\omega, \vartheta)=\tau(\vartheta)$ for all $\omega$ and $\vartheta$ ). Moreover, if $\sigma$ is a stopping time and $Z^{\sigma}: \Omega \rightarrow \mathbf{R}$ is a random variable (integrable) such that

$$
Z^{\sigma}(\omega)=Z\left(\omega, \theta_{\sigma}(\omega)\right)
$$

for some $\mathscr{F}_{\sigma} \otimes \mathscr{F}_{\infty}$-measurable random variable $Z: \Omega \times \Omega \rightarrow \mathbf{R}$ and $\omega \in \Omega$, then the strong Markov property of $X$ extends as follows:

$$
\mathbf{E}_{x}\left(Z^{\sigma} \mid \mathscr{F}_{\sigma}\right)(\omega)=\mathbf{E}_{X_{\sigma}(\omega)} Z(\omega,)
$$

for $x \in E$ and $\omega \in \Omega$. The facts (2.1)-(2.5) will be used in the proof of Theorem 2.1 below when showing that the Nash equilibrium (being attained at any two stopping times) implies the superharmonic characterization.

Recall that a measurable function $F: E \rightarrow \mathbf{R}$ is finely continuous (i.e., continuous in the fine topology) if and only if

$$
\lim _{t \downarrow 0} F\left(X_{t}\right)=F(x) \quad \mathbf{P}_{x} \text {-a.s. }
$$

for every $x \in E$. This property is further equivalent to the fact that

$$
\text { the sample path } t \mapsto F\left(X_{t}(\omega)\right) \text { is right-continuous on } \mathbf{R}_{+}
$$

for every $\omega \in \Omega \sigma N$, where $\mathbf{P}_{x}(N)=0$ for all $x \in E$. A well-known sufficient condition for a measurable function $F: E \rightarrow \mathbf{R}$ to be finely continuous is that

$$
\lim _{n \rightarrow \infty} \mathbf{E}_{x} F\left(X_{\tau_{K_{n}}}\right)=F(x)
$$

for $x \in E$ whenever $K_{1} \subseteq K_{2} \subseteq \cdots$ are compact sets in $E$ such that $\tau_{K_{n}} \downarrow 0$ $\mathbf{P}_{x}$-a.s. as $n \rightarrow \infty$, where $\tau_{K_{n}}=\inf \left\{t \geqslant 0: X_{t} \in K_{n}\right\}$ denotes the first entry time of $X$ into $K_{n}$ for $n \geqslant 1$. For more details on the facts above see, e.g., [9] and $[6]$.

2. Given finely continuous functions $G_{1}, G_{2}, G_{3}: E \rightarrow \mathbf{R}$ satisfying $G_{1} \leqslant$ $G_{3} \leqslant G_{2}$ and the following integrability condition:

$$
\mathbf{E}_{x} \sup _{t}\left|G_{i}\left(X_{t}\right)\right|<\infty \quad(i=1,2,3)
$$

for all $x \in E$, we consider the optimal stopping game, where the sup-player chooses a stopping time $\tau$ to maximize, and the inf-player chooses a stopping time $\sigma$ to minimize, the expected payoff (i.e., the payment of the inf-player to the sup-player)

$$
\begin{aligned}
\mathrm{M}_{x}(\tau, \sigma)=\mathbf{E}_{x}[ & G_{1}\left(X_{\tau}\right) I(\tau<\sigma) \\
& \left.+G_{2}\left(X_{\sigma}\right) I(\sigma<\tau)+G_{3}\left(X_{\tau}\right) I(\tau=\sigma)\right],
\end{aligned}
$$

where $X_{0}=x$ under $\mathbf{P}_{x}$. 
Define the upper value and the lower value of the game by

$$
V^{*}(x)=\inf _{\sigma} \sup _{\tau} \mathrm{M}_{x}(\tau, \sigma) \text { and } V_{*}(x)=\sup _{\tau} \inf _{\sigma} \mathrm{M}_{x}(\tau, \sigma),
$$

where the horizon $T$ (the upper bound for $\tau$ and $\sigma$ above) may be either finite or infinite. If $T<\infty$, then it will be assumed that $G_{1}\left(X_{T}\right)=G_{2}\left(X_{T}\right)=$ $G_{3}\left(X_{T}\right)$. In this case it is most interesting to consider the setting, where $X$ is a time-space process $\left(t, Y_{t}\right)$ for $t \in[0, T]$ so that $G_{i}=G_{i}(t, y)$ will be functions of both time and space for $i=1,2,3$. If $T=\infty$, then it will be assumed that

$$
\lim _{t \rightarrow \infty} G_{1}\left(X_{t}\right)=\lim _{t \rightarrow \infty} G_{2}\left(X_{t}\right) \quad \mathbf{P}_{x} \text {-a.s. }
$$

which will also be assigned as the common value $G_{3}\left(X_{\infty}\right)$ if $\tau$ and $\sigma$ are allowed to take the value $\infty$. The latter condition corresponds to «tying the rope at infinity». Another interesting example (of particular importance for the «rope» picture) is when the process $X$ is killed at the first entry time to a set (or point) so to remain at the same state forever. Although, formally speaking, this situation corresponds to the case of infinite horizon, it is also clear that the game cannot last indefinitely if the killing happens with probability one.

3. Let $F: E \rightarrow \mathbf{R}$ be a measurable function, let $C \subseteq E$ be a measurable set, and set $D=E \backslash C$. Let $\tau_{D}=\inf \left\{t \geqslant 0: X_{t} \in D\right\}$ be the first entry time of $X$ into $D$. The function $F$ is said to be superharmonic in $C$ if

$$
\mathrm{E}_{x} F\left(X_{\rho \wedge \tau_{D}}\right) \leqslant F(x)
$$

for every stopping time $\rho$ and all $x \in E$. The function $F$ is said to be subharmonic in $C$ if

$$
\mathbf{E}_{x} F\left(X_{\rho \wedge \tau_{D}}\right) \geqslant F(x)
$$

for every stopping time $\rho$ and all $x \in E$. The function $F$ is said to be harmonic in $C$ if

$$
\mathbf{E}_{x} F\left(X_{\rho \wedge \tau_{D}}\right)=F(x)
$$

for every stopping time $\rho$ and all $x \in E$. It is easily verified using the strong Markov property of $\left(X_{t \wedge \tau_{D}}\right)_{t \geqslant 0}$ and the optional sampling theorem that

$F$ is superharmonic in $C \Longleftrightarrow\left(F\left(X_{t \wedge \tau_{D}}\right)\right)_{t \geqslant 0}$ is a right-continuous

$$
\text { supermartingale, }
$$

$F$ is subharmonic in $C \Longleftrightarrow\left(F\left(X_{t \wedge \tau_{D}}\right)\right)_{t \geqslant 0}$ is a right-continuous

$$
\text { submartingale, }
$$

$F$ is harmonic in $C \Longleftrightarrow\left(F\left(X_{t \wedge \tau_{D}}\right)\right)_{t \geqslant 0}$ is a right-continuous

$$
\text { martingale }
$$

under $\mathbf{P}_{x}$ whenever $F$ is finely continuous and satisfies the integrability condition

$$
\mathbf{E}_{x} \sup _{t}\left|F\left(X_{t \wedge \tau_{D}}\right)\right|<\infty
$$


for $x \in E$. These two sufficient conditions can also be relaxed (further details will be omitted).

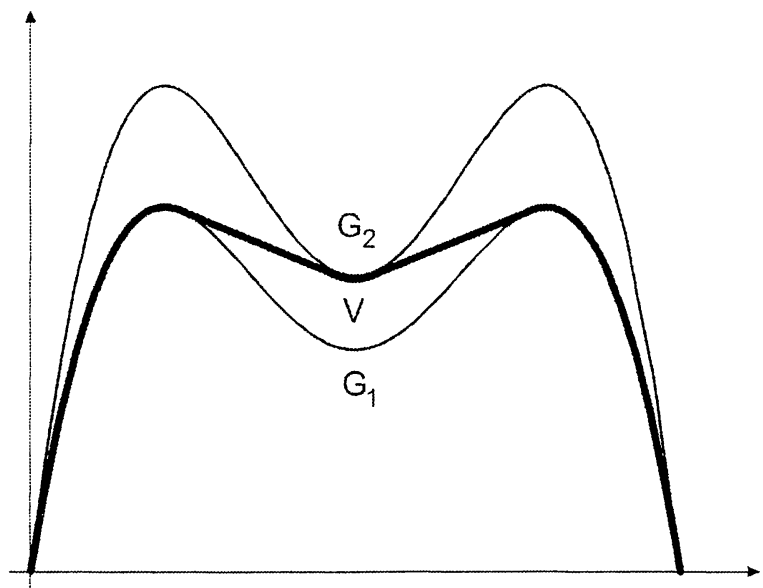

Fig. 2. Two obstacles $G_{1}$ and $G_{2}$ and the rope $V$ depicting the semiharmonic characterization of the value function in an optimal stopping game

4. Since $X$ is right-continuous we know that under (2.12) we have $V^{*}=V_{*}$ and $V:=V^{*}=V_{*}$ defines a measurable function (cf. [12, Theorem 2.1]). Let us introduce the following two classes of functions:

$\operatorname{Sup}\left[G_{1}, G_{2}\right)=\left\{F: E \rightarrow\left[G_{1}, G_{2}\right]: F\right.$ is finely continuous and

$$
\text { superharmonic in } \left.\left\{F<G_{2}\right\} \text { and }\left\{V<G_{2}\right\}\right\} \text {, }
$$

$\operatorname{Sub}\left(G_{1}, G_{2}\right]=\left\{F: E \rightarrow\left[G_{1}, G_{2}\right]: F\right.$ is finely continuous and

$$
\text { subharmonic in } \left.\left\{F>G_{1}\right\} \text { and }\left\{V>G_{1}\right\}\right\} \text {, }
$$

and let us define the following two functions:

$$
\hat{V}=\inf _{F \in \operatorname{Sup}\left[G_{1}, G_{2}\right)} F \text { and } \check{V}=\sup _{F \in \operatorname{Sub}\left[G_{1}, G_{2}\right)} F \text {. }
$$

Note that $G_{2}$ belongs to $\operatorname{Sup}\left[G_{1}, G_{2}\right)$ and that $G_{1}$ belongs to $\operatorname{Sub}\left(G_{1}, G_{2}\right]$.

We will see in the proof below that the requirement on the function $F$ from $\operatorname{Sup}\left[G_{1}, G_{2}\right)$ to be superharmonic in $\left\{F<G_{2}\right\}$ corresponds to the fact that the «rope is pulled to the ground» through the contact points with $G_{2}$, and the requirement on the same $F$ to be superharmonic in $\left\{V<G_{2}\right\}$ corresponds to the fact that the contact points with $G_{2}$ are selected among the contact points of $V$ and $G_{2}$ (the former representing a benchmark value that is invariant to the order of pulling). The analogous remark may be directed towards the two requirements on the function $F$ from $\operatorname{Sub}\left(G_{1}, G_{2}\right]$. We will also see in Example 2.2 below that neither of these requirements 
can be omitted if we are to characterize the Nash equilibrium via equality between $\hat{V}$ and $\breve{V}$ as defined in (2.22).

The main result of the paper may now be stated as follows. We note that the consequences (D)-(F) were derived in various special cases earlier in the literature (see, e.g., [18, p. 702]).

Theorem 2.1. Consider the optimal stopping game (2.11), where the strong Markov process $X$ is assumed to be right-continuous. Then $\check{V} \leqslant V_{*}=$ $V^{*} \leqslant \hat{V}$ and we have

$$
\hat{V}=\check{V} \Longleftrightarrow \text { Nash equilibrium (1.4) holds. }
$$

Moreover, in this case, setting $V:=\hat{V}=\check{V}, D_{1}=\left\{V=G_{1}\right\}, D_{2}=$ $\left\{V=G_{2}\right\}$, letting $\tau_{D_{1}}=\inf \left\{t \geqslant 0: X_{t} \in D_{1}\right\}$ denote the first entry time of $X$ into $D_{1}$, and letting $\sigma_{D_{2}}=\inf \left\{t \geqslant 0: X_{t} \in D_{2}\right\}$ denote the first entry time of $X$ into $D_{2}$, we have:

(A) The value function $V$ belongs to $\operatorname{Sup}\left[G_{1}, G_{2}\right) \cap \operatorname{Sub}\left(G_{1}, G_{2}\right]$;

(B) the first entry times $\tau_{D_{1}}$ and $\sigma_{D_{2}}$ are Nash optimal in the sense that

$$
\mathrm{M}_{x}\left(\tau, \sigma_{D_{2}}\right) \leqslant \mathrm{M}_{x}\left(\tau_{D_{1}}, \sigma_{D_{2}}\right) \leqslant \mathrm{M}_{x}\left(\tau_{D_{1}}, \sigma\right)
$$

for all stopping times $\tau$ and $\sigma$, and all $x \in E$;

(C) if $\tau_{*}$ and $\sigma_{*}$ are Nash optimal stopping times, then

$$
\tau_{D_{1}} \leqslant \tau_{*} \quad \mathbf{P}_{x} \text {-a.s. and } \sigma_{D_{2}} \leqslant \sigma_{*} \quad \mathbf{P}_{x} \text {-a.s. }
$$

for all $x \in E$;

(D) the value function $V$ is subharmonic in $C_{1}=\left\{V>G_{1}\right\}$, i.e., the stopped process $\left(V\left(X_{t \wedge \tau_{D_{1}}}\right)\right)_{t \geqslant 0}$ is a right-continuous submartingale;

(E) the value function $V$ is superharmonic in $C_{2}=\left\{V<G_{2}\right\}$, i.e., the stopped process $\left(V\left(X_{t \wedge \sigma_{D_{2}}}\right)\right)_{t \geqslant 0}$ is a right-continuous supermartingale;

$(\mathrm{F})$ the value function $V$ is harmonic in $C_{1} \cap C_{2}$, i.e., the stopped process $\left(V\left(X_{t \wedge \tau_{D_{1}} \wedge \sigma_{D_{2}}}\right)\right)_{t \geqslant 0}$ is a right-continuous martingale.

In particular, if the strong Markov process $X$ is right-continuous and left-continuous over stopping times, then (2.23) and (A)-(F) are satisfied.

$\mathrm{P}$ r o o f. Since $X$ is right-continuous, we know that $V^{*}=V_{*}$ and $V:=$ $V^{*}=V_{*}$ defines a measurable function (recall the text stated prior to $(2.20)$ above).

1) We first show that the value function $V$ is finely continuous. For this, take any stopping times $\rho_{n}$ and $\rho$ such that $\rho_{n} \downarrow \rho$ as $n \rightarrow \infty$ and recall from (2.8) that it is enough to show that $\mathbf{E}_{x} V\left(X_{\rho_{n}}\right) \rightarrow \mathbf{E}_{x} V\left(X_{\rho}\right)$ as $n \rightarrow \infty$. This will be done in three steps as follows.

Firstly, let us recall that we have

$$
\mathbf{E}_{x} V\left(X_{\rho}\right)=\inf _{\sigma \geqslant \rho} \sup _{\tau \geqslant \rho} M_{x}(\tau, \sigma)=\sup _{\tau \geqslant \rho} \inf _{\sigma \geqslant \rho} M_{x}(\tau, \sigma)
$$


for all $x \in E$. This is a consequence of the strong Markov property of $X$ and the fact that the underlying families of random variables are downwards and upwards directed (for further details see [12] and [27]).

Secondly, for any stopping time $\sigma>\rho_{n}$ we find that

$$
\begin{aligned}
\mathrm{M}_{x}(\tau, \sigma) \leqslant & \mathbf{E}_{x}\left(\left[G_{1}\left(X_{\tau}\right) I(\tau<\sigma)+G_{2}\left(X_{\sigma}\right) I(\sigma<\tau)\right.\right. \\
& \left.\left.+G_{3}\left(X_{\tau}\right) I(\tau=\sigma)\right] I\left(\tau<\rho_{n}\right)\right) \\
+ & \mathbf{E}_{x}\left(\left[G_{1}\left(X_{\tau \vee \rho_{n}}\right) I\left(\tau \vee \rho_{n}<\sigma\right)+G_{2}\left(X_{\sigma}\right) I\left(\sigma<\tau \vee \rho_{n}\right)\right.\right. \\
& \left.\left.\quad+G_{3}\left(X_{\sigma}\right) I\left(\sigma=\tau \vee \rho_{n}\right)\right] I\left(\tau \geqslant \rho_{n}\right)\right) \\
= & \mathbf{E}_{x}\left[G_{1}\left(X_{\tau}\right) I\left(\tau<\rho_{n}\right)\right] \\
& +\mathbf{E}_{x}\left(G_{1}\left(X_{\tau \vee \rho_{n}}\right) I\left(\tau \vee \rho_{n}<\sigma\right)+G_{2}\left(X_{\sigma}\right) I\left(\sigma<\tau \vee \rho_{n}\right)\right. \\
& \left.\quad+G_{3}\left(X_{\sigma}\right) I\left(\sigma=\tau \vee \rho_{n}\right)\right) \\
& \quad-\mathbf{E}_{x}\left(\left[G_{1}\left(X_{\tau \vee \rho_{n}}\right) I\left(\tau \vee \rho_{n}<\sigma\right)+G_{2}\left(X_{\sigma}\right) I\left(\sigma<\tau \vee \rho_{n}\right)\right.\right. \\
& \left.\left.\quad+G_{3}\left(X_{\sigma}\right) I\left(\sigma=\tau \vee \rho_{n}\right)\right] I\left(\tau<\rho_{n}\right)\right) \\
= & \mathbf{E}_{x}\left[G_{1}\left(X_{\tau}\right) I\left(\tau<\rho_{n}\right)-G_{1}\left(X_{\rho_{n}}\right) I\left(\tau<\rho_{n}\right)\right]+\mathrm{M}_{x}\left(\tau \vee \rho_{n}, \sigma\right) \\
= & \mathbf{E}_{x}\left[G_{1}\left(X_{\tau \wedge \rho_{n}}\right)-G_{1}\left(X_{\rho_{n}}\right)\right]+\mathrm{M}_{x}\left(\tau \vee \rho_{n}, \sigma\right) .
\end{aligned}
$$

From (2.24) and (2.25) it follows that

$$
\begin{aligned}
\mathbf{E}_{x} V\left(X_{\rho}\right) & \leqslant \mathbf{E}_{x} \sup _{\rho \leqslant t \leqslant \rho_{n}}\left|G_{1}\left(X_{t}\right)-G_{1}\left(X_{\rho_{n}}\right)\right|+\inf _{\sigma>\rho_{n}} \sup _{\tau \geqslant \rho_{n}} \mathrm{M}_{x}(\tau, \sigma) \\
& =\mathbf{E}_{x} \sup _{\rho \leqslant t \leqslant \rho_{n}}\left|G_{1}\left(X_{t}\right)-G_{1}\left(X_{\rho_{n}}\right)\right|+\mathbf{E}_{x} V\left(X_{\rho_{n}}\right)
\end{aligned}
$$

for all $n \geqslant 1$, where the equality can be justified as follows. For stopping times $\tau, \sigma$, and $\sigma_{n}$ such that $\sigma \leqslant \sigma_{n}$ we have

$$
\begin{aligned}
\mathrm{M}_{x}(\tau, \sigma) & -\mathrm{M}_{x}\left(\tau, \sigma_{n}\right) \\
=\mathbf{E}_{x}[ & G_{1}\left(X_{\tau}\right) I(\tau<\sigma)+G_{2}\left(X_{\sigma}\right) I(\sigma<\tau)+G_{3}\left(X_{\tau}\right) I\left(\tau=\sigma, \tau \neq \sigma_{n}\right) \\
& -G_{1}\left(X_{\tau}\right) I\left(\tau<\sigma_{n}\right)-G_{2}\left(X_{\sigma_{n}}\right) I\left(\sigma_{n}<\tau\right) \\
& \left.-G_{3}\left(X_{\tau}\right) I\left(\tau=\sigma_{n}, \tau \neq \sigma\right)\right] \\
\geqslant \mathbf{E}_{x}( & G_{1}\left(X_{\tau}\right)\left[I(\tau<\sigma)+I\left(\tau=\sigma, \tau \neq \sigma_{n}\right)\right]+G_{2}\left(X_{\sigma}\right) I(\sigma<\tau) \\
& \left.-G_{1}\left(X_{\tau}\right) I\left(\tau<\sigma_{n}\right)-G_{2}\left(X_{\sigma_{n}}\right)\left[I\left(\sigma_{n}<\tau\right)+I\left(\tau=\sigma_{n}, \tau \neq \sigma\right)\right]\right) \\
=\mathbf{E}_{x}( & {\left[G_{2}\left(X_{\sigma}\right)-G_{2}\left(X_{\sigma_{n}}\right)\right] I(\sigma<\tau) } \\
& +G_{1}\left(X_{\tau}\right)\left[I(\tau<\sigma)-I\left(\tau<\sigma_{n}\right)+I\left(\tau=\sigma, \tau \neq \sigma_{n}\right)\right] \\
& \left.+G_{2}\left(X_{\sigma_{n}}\right)\left[I(\sigma<\tau)-I\left(\sigma_{n}<\tau\right)-I\left(\tau=\sigma_{n}, \tau \neq \sigma\right)\right]\right)
\end{aligned}
$$




$$
\begin{aligned}
=\mathbf{E}_{x} & \left(\left[G_{2}\left(X_{\sigma}\right)-G_{2}\left(X_{\sigma_{n}}\right)\right] I(\sigma<\tau)\right. \\
& \left.+\left[G_{2}\left(X_{\sigma_{n}}\right)-G_{1}\left(X_{\tau}\right)\right] I\left(\sigma<\tau<\sigma_{n}\right)\right) .
\end{aligned}
$$

If $\left\{\sigma_{m}: m \geqslant 1\right\}$ is taken to be a strictly decreasing sequence of (discrete) stopping times such that $\sigma_{m} \downarrow \sigma$ as $m \rightarrow \infty$, then by (2.7) and (2.9) we see that the right-hand side of (2.27) tends to zero uniformly over all $\tau$. It follows that

$$
\sup _{\tau \geqslant \rho_{n}} M_{x}(\tau, \sigma) \geqslant \limsup _{m \rightarrow \infty} \sup _{\tau \geqslant \rho_{n}} M_{x}\left(\tau, \sigma_{m}\right) \geqslant \inf _{\sigma>\rho_{n}} \sup _{\tau \geqslant \rho_{n}} M_{x}(\tau, \sigma)
$$

for all $\sigma \geqslant \rho_{n}$. Taking the infimum over all $\sigma \geqslant \rho_{n}$ we find that the equality in (2.26) holds as claimed.

Thirdly, letting $n \rightarrow \infty$ in (2.26) and using (2.7) with (2.9) we find that

$$
\mathbf{E}_{x} V\left(X_{\rho}\right) \leqslant \liminf _{n \rightarrow \infty} \mathbf{E}_{x} V\left(X_{\rho_{n}}\right) .
$$

Applying the preceding conclusion to the optimal stopping game with the gain functions $\widetilde{G}_{1}:=-G_{2}, \widetilde{G}_{2}:=-G_{1}$, and $\widetilde{G}_{3}:=-G_{3}$, it follows that the value function $\widetilde{V}=-V$ satisfies inequality (2.29) which is the same as

$$
\mathbf{E}_{x} V\left(X_{\rho}\right) \geqslant \limsup _{n \rightarrow \infty} \mathbf{E}_{x} V\left(X_{\rho_{n}}\right) .
$$

Combining (2.29) and (2.30) we see that $\mathbf{E}_{x} V\left(X_{\rho_{n}}\right) \rightarrow \mathbf{E}_{x} V\left(X_{\rho}\right)$ as $n \rightarrow \infty$, and thus $V$ is finely continuous as claimed.

2) To derive (2.23) we will first show that

$$
\check{V} \leqslant V_{*} \leqslant V^{*} \leqslant \hat{V}
$$

on $E$. For this, take an arbitrary function $F$ from $\operatorname{Sup}\left[G_{1}, G_{2}\right)$, set $D_{2, F}=$ $\left\{F=G_{2}\right\}$, and let $\sigma_{D_{2, F}}=\inf \left\{t \geqslant 0: X_{t} \in D_{2, F}\right\}$ denote the first entry time of $X$ into $D_{2, F}$. Since both $F$ and $G_{2}$ are finely continuous, it is easily seen using (2.7) that $F\left(X_{\sigma_{2, F}}\right)=G_{2}\left(X_{\sigma_{2, F}}\right)$ when $\sigma_{D_{2, F}}<\infty$, and (2.12) implies that the previous identity also holds when $\sigma_{D_{2, F}}=\infty$. Hence for any stopping time $\tau$ we have

$$
\begin{aligned}
F\left(X_{\tau \wedge \sigma_{D_{2, F}}}\right)= & F\left(X_{\tau}\right) I\left(\tau<\sigma_{D_{2, F}}\right)+G_{2}\left(X_{\sigma_{D_{2, F}}}\right) I\left(\sigma_{D_{2, F}} \leqslant \tau\right) \\
\geqslant & G_{1}\left(X_{\tau}\right) I\left(\tau<\sigma_{D_{2, F}}\right)+G_{2}\left(X_{\sigma_{D_{2, F}}}\right) I\left(\sigma_{D_{2, F}}<\tau\right) \\
& +G_{3}\left(X_{\tau}\right) I\left(\tau=\sigma_{D_{2, F}}\right) .
\end{aligned}
$$

Since $F$ is superharmonic in $E \backslash D_{2, F}$, it follows that

$$
F(x) \geqslant \mathbf{E}_{x} F\left(X_{\tau \wedge \sigma_{D_{2, F}}}\right) \geqslant \mathrm{M}_{x}\left(\tau, \sigma_{D_{2, F}}\right)
$$

for all stopping times $\tau$ and all $x \in E$. Taking the supremum over all $\tau$, and then the infimum over all $\sigma$, we get

$$
F(x) \geqslant \sup _{\tau} \mathrm{M}_{x}\left(\tau, \sigma_{D_{2, F}}\right) \geqslant \inf _{\sigma} \sup _{\tau} \mathrm{M}_{x}(\tau, \sigma)=V^{*}(x)
$$


for all $x \in E$. Taking the infimum over all $F$ in $\operatorname{Sup}\left[G_{1}, G_{2}\right)$ we can then conclude that $\hat{V}(x) \geqslant V^{*}(x)$ for all $x \in E$. The inequality $\check{V} \leqslant V_{*}$ can be proved analogously (or follows by symmetry). Combining the two inequalities we get (2.31) as claimed.

3) Let us now show that $\hat{V}=\check{V}$ in (2.23) implies that the Nash equilibrium (1.4) holds. For this, recall that $V:=V^{*}=V_{*}$, set $D_{2}=\left\{V=G_{2}\right\}$, and let $\sigma_{D_{2}}=\inf \left\{t \geqslant 0: X_{t} \in D_{2}\right\}$ denote the first entry time of $X$ into $D_{2}$. Then as above since both $V$ and $G_{2}$ are finely continuous, it is easily seen using (2.7) that $V\left(X_{\sigma_{D_{2}}}\right)=G_{2}\left(X_{\sigma_{D_{2}}}\right)$, when $\sigma_{D_{2}}<\infty$, and (2.12) implies that the previous identity also holds when $\sigma_{D_{2}}=\infty$. Hence taking any $F \in \operatorname{Sup}\left[G_{1}, G_{2}\right)$, recalling that $F$ is superharmonic in $E \backslash D_{2}$ and $F \geqslant V \geqslant G_{1}$, it follows as in (2.32) and (2.33) above that for any stopping time $\tau$ we have

$$
\begin{aligned}
F(x) \geqslant & \mathbf{E}_{x} F\left(X_{\tau \wedge \sigma_{D_{2}}}\right) \geqslant \mathbf{E}_{x} V\left(X_{\tau \wedge \sigma_{D_{2}}}\right) \\
= & \mathbf{E}_{x}\left[V\left(X_{\tau}\right) I\left(\tau<\sigma_{D_{2}}\right)+G_{2}\left(X_{\sigma_{D_{2}}}\right) I\left(\sigma_{D_{2}} \leqslant \tau\right)\right] \\
\geqslant & \mathbf{E}_{x}\left[G_{1}\left(X_{\tau}\right) I\left(\tau<\sigma_{D_{2}}\right)+G_{2}\left(X_{\sigma_{D_{2}}}\right) I\left(\sigma_{D_{2}}<\tau\right)\right. \\
\quad \quad & \left.\quad G_{3}\left(X_{\tau}\right) I\left(\tau=\sigma_{D_{2}}\right)\right]=\mathrm{M}_{x}\left(\tau, \sigma_{D_{2}}\right)
\end{aligned}
$$

for all $x \in E$. Taking the infimum over all $F$ in $\operatorname{Sup}\left[G_{1}, G_{2}\right)$ we get

$$
\hat{V}(x) \geqslant \mathrm{M}_{x}\left(\tau, \sigma_{D_{2}}\right)
$$

for all stopping times $\tau$ and all $x \in E$. It can be proved analogously (or follows by symmetry) that the following inequality holds

$$
\check{V}(x) \leqslant \mathrm{M}_{x}\left(\tau_{D_{1}}, \sigma\right)
$$

for all stopping times $\sigma$ and all $x \in E$, where $D_{1}=\left\{V=G_{1}\right\}$ and $\tau_{D_{1}}=$ $\inf \left\{t \geqslant 0: X_{t} \in D_{1}\right\}$ denotes the first entry time of $X$ into $D_{1}$. Combining (2.36) and (2.37) with the fact that $V=\hat{V}=\breve{V}$ (which follows by (2.31) and the hypothesis) we see that

$$
\mathrm{M}_{x}\left(\tau, \sigma_{D_{2}}\right) \leqslant V(x) \leqslant \mathrm{M}_{x}\left(\tau_{D_{1}}, \sigma\right)
$$

for all stopping times $\tau$ and $\sigma$, and for all $x \in E$. This shows that the Nash equilibrium (1.4) holds with $\tau_{*}=\tau_{D_{1}}$ and $\sigma_{*}=\sigma_{D_{2}}$. Moreover, taking the infimum over all $F \in \operatorname{Sup}\left[G_{1}, G_{2}\right)$ in $(2.35)$, we see from the first two inequalities that $V$ is superharmonic in $E \backslash D_{2}$ and thus belongs to $\operatorname{Sup}\left[G_{1}, G_{2}\right)$. The fact that $V$ belongs to $\operatorname{Sub}\left(G_{1}, G_{2}\right]$ can be proved analogously (or follows by symmetry). This establishes the assertion (A) and hence the assertions (D) $-(\mathrm{F})$ follow by $(2.16)-(2.18)$. This completes the first part of the proof.

4) We now show that the assertion (C) holds. For this, let us assume that the Nash equilibrium (1.4) holds with some stopping times $\tau_{*}$ and $\sigma_{*}$. 
We will first verify that (1.4) implies that

$$
\begin{aligned}
\mathbf{E}_{x}\left[G_{1}\left(X_{\tau_{*}}\right) I\left(\tau_{*}=\sigma_{*}\right)\right] & =\mathbf{E}_{x}\left[G_{3}\left(X_{\tau_{*}}\right) I\left(\tau_{*}=\sigma_{*}\right)\right] \\
& =\mathbf{E}_{x}\left[G_{2}\left(X_{\tau_{*}}\right) I\left(\tau_{*}=\sigma_{*}\right)\right]
\end{aligned}
$$

for all $x \in E$. Indeed, if we suppose that the second identity in (2.39) is not satisfied, i.e.,

$$
\mathbf{E}_{x}\left[G_{2}\left(X_{\tau_{*}}\right) I\left(\tau_{*}=\sigma_{*}\right)\right]>\mathbf{E}_{x}\left[G_{3}\left(X_{\tau_{*}}\right) I\left(\tau_{*}=\sigma_{*}\right)\right],
$$

we can set $\tau_{*}^{\varepsilon}=\left(\tau_{*}+\varepsilon\right) 1_{A}+\tau_{*} 1_{A^{c}}$, where $A=\left\{\tau_{*}=\sigma_{*}\right\}$ and conclude that $\tau_{*}^{\varepsilon}$ is a stopping time, since $A \in \mathscr{F}_{\tau_{*} \wedge \sigma_{*}} \subseteq \mathscr{F}_{\tau_{*}}$. Note that there is no restriction to assume that $\tau_{*}<\infty$ and $\sigma_{*}<\infty$ on $A$ due to (2.12). Likewise, if the horizon $T$ is finite, then $\tau_{*}+\varepsilon$ in the definition of $\tau_{*}^{\varepsilon}$ above should be replaced by $\left(\tau_{*}+\varepsilon\right) \wedge T$ and the remaining part of the proof can be carried out in exactly the same way. Circumventing these technicalities we find that

$$
\begin{aligned}
\mathrm{M}_{x}\left(\tau_{*}^{\varepsilon}, \sigma_{*}\right)= & \mathbf{E}_{x}\left[G_{1}\left(X_{\tau_{*}}\right) I\left(\tau_{*}^{\varepsilon}<\sigma_{*}\right)+G_{2}\left(X_{\sigma_{*}}\right) I\left(\sigma_{*}<\tau_{*}^{\varepsilon}\right)\right. \\
& \left.\quad+G_{3}\left(X_{\tau_{*}^{\varepsilon}}\right) I\left(\tau_{*}^{\varepsilon}=\sigma_{*}\right)\right] \\
= & \mathbf{E}_{x}\left[G_{1}\left(X_{\tau_{*}}\right) I\left(\tau_{*}<\sigma_{*}\right)+G_{2}\left(X_{\sigma_{*}}\right)\left(1_{A}+I\left(\sigma_{*}<\tau_{*}\right)\right)\right] \\
= & \mathbf{E}_{x}\left[G_{1}\left(X_{\tau_{*}}\right) I\left(\tau_{*}<\sigma_{*}\right)+G_{2}\left(X_{\sigma_{*}}\right) I\left(\sigma_{*} \leqslant \tau_{*}\right)\right] \\
> & \mathbf{E}_{x}\left[G_{1}\left(X_{\tau_{*}}\right) I\left(\tau_{*}<\sigma_{*}\right)+G_{2}\left(X_{\sigma_{*}}\right) I\left(\sigma_{*}<\tau_{*}\right)\right. \\
\quad & \left.\quad G_{3}\left(X_{\sigma_{*}}\right) I\left(\sigma_{*}=\tau_{*}\right)\right]=\mathrm{M}_{x}\left(\tau_{*}, \sigma_{*}\right)
\end{aligned}
$$

upon using (2.40). Since the strict inequality in (2.41) violates the first inequality in (1.4), we see that (2.40) must be false. Hence the second identity in (2.39) holds as claimed. The first identity in (2.39) can be proved analogously (or follows by symmetry).

Let us now show that $\tau_{D_{1}} \leqslant \tau_{*} \mathbf{P}_{x}$-a.s. for each $x \in E$ given and fixed. For this, note that by (1.4) and the first identity in (2.39) we have

$$
V(x)=\mathrm{M}_{x}\left(\tau_{*}, \sigma_{*}\right)=\mathbf{E}_{x}\left[G_{1}\left(X_{\tau_{*}}\right) I\left(\tau_{*} \leqslant \sigma_{*}\right)+G_{2}\left(X_{\sigma_{*}}\right) I\left(\sigma_{*}<\tau_{*}\right)\right] .
$$

Hence by the strong Markov property of $X$ we get

$$
\begin{aligned}
\mathbf{E}_{x} V\left(X_{\tau_{*}}\right)= & \mathbf{E}_{x} M_{X_{\tau_{*}}}\left(\tau_{*}, \sigma_{*}\right) \\
= & \mathbf{E}_{x}\left[\mathbf { E } _ { x } \left[G_{1}\left(X_{\tau_{*}}\right) \circ \theta_{\tau_{*}} I\left(\tau_{*} \circ \theta_{\tau_{*}} \leqslant \sigma_{*} \circ \theta_{\tau_{*}}\right)\right.\right. \\
& \left.\left.\quad+G_{2}\left(X_{\sigma_{*}}\right) \circ \theta_{\tau_{*}} I\left(\sigma_{*} \circ \theta_{\tau_{*}}<\tau_{*} \circ \theta_{\tau_{*}}\right) \mid \mathscr{F}_{\tau_{*}}\right]\right] \\
= & \mathbf{E}_{x} G_{1}\left(X_{\tau_{*}}\right)
\end{aligned}
$$

upon using that $G_{1}\left(X_{\tau_{*}}\right) \circ \theta_{\tau_{*}}=G_{1}\left(X_{\tau_{*}+\tau_{*} \circ \theta_{\tau_{*}}}\right)=G_{1}\left(X_{\tau_{*}}\right)$, since $\tau_{*} \circ \theta_{\tau_{*}}=0$. From (2.43) we see that $V\left(X_{\tau_{*}}\right)=G_{1}\left(X_{\tau_{*}}\right) \mathbf{P}_{x}$-a.s. and hence $\tau_{D_{1}} \leqslant \tau_{*}$ 
$\mathbf{P}_{x}$-a.s. by definition of $\tau_{D_{1}}$. The inequality $\sigma_{D_{2}} \leqslant \sigma_{*} \mathbf{P}_{x}$-a.s. can be proved analogously (or follows by symmetry).

5) Let us now show that the reverse implication in (2.23) holds, i.e., let us assume that the Nash equilibrium (1.4) holds with some stopping times $\tau_{*}$ and $\sigma_{*}$, and let us show that this fact implies that $\hat{V}=\check{V}$. For this, in view of $(2.31)$ it is enough to show that $V$ belongs to both $\operatorname{Sup}\left[G_{1}, G_{2}\right)$ and $\operatorname{Sub}\left(G_{1}, G_{2}\right]$. To show that $V$ belongs to $\operatorname{Sup}\left[G_{1}, G_{2}\right)$ it is sufficient to take any stopping time $\tau$ and for a given and fixed $x \in E$ show that

$$
\mathbf{E}_{x} V\left(X_{\rho}\right) \leqslant V(x)
$$

where $\rho=\tau \wedge \sigma_{D_{2}}$ and $\sigma_{D_{2}}=\inf \left\{t \geqslant 0: X_{t} \in D_{2}\right\}$ denotes the first entry time of $X$ into $D_{2}$. To derive (2.44) let us first assume that $\sigma_{*}$ is the first entry time of $X$ into a Borel set (this assumption is only made for the reasons of comparison with the more general proof below). Since by (C) we have $\rho \leqslant \sigma_{*}$, it follows that $\sigma_{*}=\rho+\sigma_{*} \circ \theta_{\rho}$ (recall the sentence following (2.3) above). Hence by the strong Markov property of $X$ we get

$$
\begin{aligned}
& \mathbf{E}_{x} V\left(X_{\rho}\right)= \mathbf{E}_{x} M_{X_{\rho}}\left(\tau_{*}, \sigma_{*}\right) \\
&=\mathbf{E}_{x}\left[\mathbf{E}_{x}[\right. G_{1}\left(X_{\tau_{*}}\right) \circ \theta_{\rho} I\left(\tau_{*} \circ \theta_{\rho}<\sigma_{*} \circ \theta_{\rho}\right) \\
& \quad \quad G_{2}\left(X_{\sigma_{*}}\right) \circ \theta_{\rho} I\left(\sigma_{*} \circ \theta_{\rho}<\tau_{*} \circ \theta_{\rho}\right) \\
& \quad\left.\left.\quad G_{3}\left(X_{\tau_{*}}\right) \circ \theta_{\rho} I\left(\tau_{*} \circ \theta_{\rho}=\sigma_{*} \circ \theta_{\rho}\right) \mid \mathscr{F}_{\rho}\right]\right] \\
&=\mathbf{E}_{x}\left[G_{1}\left(X_{\tau_{*}^{\prime}}\right) I\left(\tau_{*}^{\prime}<\sigma_{*}\right)+G_{2}\left(X_{\sigma_{*}}\right) I\left(\sigma_{*}<\tau_{*}^{\prime}\right)\right. \\
&\left.\quad+G_{3}\left(X_{\tau_{*}^{\prime}}\right) I\left(\tau_{*}^{\prime}=\sigma_{*}\right)\right]=\mathrm{M}_{x}\left(\tau_{*}^{\prime}, \sigma_{*}\right) \leqslant V(x),
\end{aligned}
$$

where $\tau_{*}^{\prime}=\rho+\tau_{*} \circ \theta_{\rho}$ is a stopping time and the final inequality follows by the first inequality in (1.4). This establishes (2.44) when $\sigma_{*}$ is the first entry time of $X$ into a Borel set.

Let us now show that (2.44) also holds when $\sigma_{*}$ is an arbitrary stopping time. Since by $(\mathrm{C})$ we have $\rho \leqslant \sigma_{*}$, it follows by $(2.1)-(2.3)$ that

$$
\sigma_{*}(\omega)=\rho(\omega)+\sigma_{*}^{\rho}\left(\omega, \theta_{\rho}(\omega)\right)
$$

where the function $\sigma_{*}^{\rho}: \Omega \times \Omega \rightarrow[0, \infty]$ is $\mathscr{F}_{\sigma} \otimes \mathscr{F}_{\infty}$-measurable and $\vartheta \mapsto \sigma_{*}^{\rho}(\omega, \vartheta)$ is a stopping time for every $\omega \in \Omega$. Hence by the second inequality in (1.4) we have

$$
V(x) \leqslant M_{x}\left(\tau_{*}, \sigma_{*}^{\rho}(\omega,)\right)
$$

for all $x \in E$ and each $\omega \in \Omega$ given and fixed. Setting $x=X_{\rho}(\omega)$ in $(2.46)$ we get

$$
V\left(X_{\rho}(\omega)\right) \leqslant \mathrm{M}_{X_{\rho}(\omega)}\left(\tau_{*}, \sigma_{*}^{\rho}(\omega,)\right)
$$


for all $\omega \in \Omega$. Taking $\mathbf{E}_{x}$ on both sides in (2.47) with respect to $\omega$ and applying the extended strong Markov property (2.5) we obtain

$$
\begin{aligned}
\mathbf{E}_{x} V\left(X_{\rho}(\omega)\right)= & \mathbf{E}_{x} M_{X_{\rho}(\omega)}\left(\tau_{*}, \sigma_{*}^{\rho}(\omega,)\right) \\
=\mathbf{E}_{x}\left[\mathbf{E}_{x}[\right. & G_{1}\left(X_{\tau_{*}}\right) \circ \theta_{\rho} I\left(\tau_{*} \circ \theta_{\rho}<\sigma_{*}^{\rho}\left(\theta_{\rho}\right)\right) \\
\quad & \quad G_{2}\left(X_{\sigma_{*}(, .)}\right) \circ \theta_{\rho} I\left(\sigma_{*}^{\rho}\left(, \theta_{\rho}\right)<\tau_{*} \circ \theta_{\rho}\right) \\
\quad & \left.\left.\quad G_{3}\left(X_{\tau_{*}}\right) \circ \theta_{\rho} I\left(\tau_{*} \circ \theta_{\rho}<\sigma_{*}^{\rho}\left(, \theta_{\rho}\right)\right) \mid \mathscr{F}_{\rho}\right](\omega)\right] \\
=\mathbf{E}_{x}[ & G_{1}\left(\tau_{*}^{\prime}\right) I\left(\tau_{*}^{\prime}<\sigma_{*}\right)+G_{2}\left(X_{\sigma_{*}}\right) I\left(\sigma_{*}<\tau_{*}^{\prime}\right) \\
\quad & \left.\quad G_{3}\left(X_{\tau_{*}^{\prime}}\right) I\left(\tau_{*}^{\prime}=\sigma_{*}\right)\right]=\mathrm{M}_{x}\left(\tau_{*}^{\prime}, \sigma_{*}\right) \leqslant V(x),
\end{aligned}
$$

where $\tau_{*}^{\prime}=\rho+\tau_{*} \circ \theta_{\rho}$ is a stopping time and the final inequality follows by the first inequality in (1.4). This establishes (2.44) when $\sigma_{*}$ is an arbitrary stopping time and hence $V$ belongs to $\operatorname{Sup}\left[G_{1}, G_{2}\right)$ as claimed. The fact that $V$ belongs to $\operatorname{Sub}\left(G_{1}, G_{2}\right]$ can be proved analogously (or follows by symmetry). Since $V$ is shown to belong to both $\operatorname{Sup}\left[G_{1}, G_{2}\right)$ and $\operatorname{Sub}\left(G_{1}, G_{2}\right]$, it follows by (2.31) that $\hat{V}=\check{V}$ and the proof is complete.

The following example shows that neither of the requirements in the definitions (2.20) and (2.21) can be omitted if we are to characterize the Nash equilibrium via equality between the smallest superharmonic and the largest subharmonic function.

E x a m p le 2.1. Let the state space $E$ of the process $X$ be $[-1,1]$. If $X$ starts at $x \in(-1,1)$ let $X$ be a standard Brownian motion $B$ until it hits either -1 or 1 ; if $X$ hits -1 before 1 let $X$ be killed and remain at -1 forever; if $X$ hits 1 before -1 let $X$ start afresh from 0 as an independent copy of $B$; and so on. If $X$ starts at $x \in\{-1,1\}$ let $X$ stay at the same $x$ for the rest of time. It follows that $X$ is a right-continuous strong Markov process which is not left-continuous over stopping times. Indeed, if we consider the first hitting time $\rho_{\varepsilon}$ of $X$ to $1-\varepsilon$ under $\mathbf{P}_{x}$ for $x \in(-1,1)$ given and fixed, then $\rho_{\varepsilon} \uparrow \rho$ as $\varepsilon \downarrow 0$ so that $\rho$ is a stopping time, however, the value $X_{\rho_{\varepsilon}}=1-\varepsilon$ does not converge to $X_{\rho} \in\{-1,0\}$ as $\varepsilon \downarrow 0$ on the set $\{\rho<\infty\}$ which has strictly positive $\mathbf{P}_{x}$-measure, implying the claim.

Let $G_{1}(x)=x(x+1)-1$ and $G_{2}(x)=-x(x-1)+1$ for $x \in[-1,1]$, and let $G_{3}$ be equal to $G_{1}$ on $[-1,1]$. Note that $G_{i}(-1)=-1$ and $G_{i}(1)=1$ for $i=1,2,3$. Note also that $\lim _{t \rightarrow \infty} G_{i}\left(X_{t}\right)=G_{i}(-1)=-1$ so that $G_{i}\left(X_{\infty}\right)$ is naturally set to be equal to -1 under $\mathbf{P}_{x}$ for $x \in[-1,1)$ and $i=1,2,3$. On the other hand, under $\mathbf{P}_{x} 1$ we clearly have $G_{i}\left(X_{\infty}\right):=\lim _{t \rightarrow \infty} G_{i}\left(X_{t}\right)=$ $G_{i}(1)=1$ for $i=1,2,3$. It is then easily seen that $V^{*}(x)=V_{*}(x)=x$ for all $x \in[-1,1]$ with $\tau_{\varepsilon}=\inf \left\{t \geqslant 0: X_{t} \leqslant a_{\varepsilon}^{1}\right.$ or $\left.X_{t} \geqslant b_{\varepsilon}^{1}\right\}$ (where $a_{\varepsilon}^{1}<b_{\varepsilon}^{1}$ satisfy $G_{1}\left(a_{\varepsilon}^{1}\right)=a_{\varepsilon}^{1}-\varepsilon$ and $\left.G_{1}\left(b_{\varepsilon}^{1}\right)=b_{\varepsilon}^{1}-\varepsilon\right)$ and $\sigma_{\varepsilon}=\inf \left\{t \geqslant 0: X_{t} \leqslant a_{\varepsilon}^{2}\right.$ or $\left.X_{t} \geqslant b_{\varepsilon}^{2}\right\}$ (where $a_{\varepsilon}^{2}<b_{\varepsilon}^{2}$ satisfy $G_{2}\left(a_{\varepsilon}^{2}\right)=a_{\varepsilon}^{2}+\varepsilon$ and $G_{2}\left(b_{\varepsilon}^{2}\right)=b_{\varepsilon}^{2}+\varepsilon$ ) being approximate 
stopping times satisfying $\mathrm{M}_{x}\left(\tau, \sigma_{\varepsilon}\right)-\varepsilon \leqslant V_{*}(x) \leq V^{*}(x) \leqslant \mathrm{M}_{x}\left(\tau_{\varepsilon}, \sigma\right)+\varepsilon$ for all stopping times $\tau$ and $\sigma$, all $x \in[-1,1]$, and all $\varepsilon>0$. For this, note that $X$ is a standard Brownian motion before hitting either $a_{\varepsilon}^{i}$ or $b_{\varepsilon}^{i}$ for $i=1,2$, respectively, and taking first the infimum over all $\sigma$ and then the supremum over all $\tau$ in the final inequality above, one finds that $V^{*} \leqslant V_{*}+\varepsilon$, and hence $V^{*}=V_{*}$ follows as well. Thus the Stackelberg equilibrium (1.3) holds with $V(x)=x$ for all $x \in[-1,1]$. It is clear, however, that the Nash equilibrium fails as it is impossible to find stopping times $\tau_{*}$ and $\sigma_{*}$ satisfying (1.4) above. Note that the natural candidates $\tau \equiv \infty$ and $\sigma \equiv \infty$ are ruled out, since $\mathrm{M}_{x}(\infty, \infty)=-1$ for $x \in[-1,1)$ and $\mathrm{M}_{x}(\infty, \infty)=1$ for $x=1$, which differs from $V(x)=x$ when $x \in[-1,1)$.

Now consider a function $F_{\varepsilon}:[-1,1] \rightarrow \mathbf{R}$ that is linear on $[-1+\varepsilon$, $1-\varepsilon]$ and satisfies $F_{\varepsilon}(x)=G_{2}(x)$ for $x \in[-1,-1+\varepsilon] \cup[1-\varepsilon, 1]$ and $\varepsilon>0$. Clearly each such $F_{\varepsilon}$ satisfies $G_{1} \leqslant F_{\varepsilon} \leqslant G_{2}$ on $[-1,1]$ and is (finely) continuous and superharmonic in $\left\{F_{\varepsilon}<G_{2}\right\}$. Taking the infimum over all $F_{\varepsilon}$ as in $(2.22)$ above when $\varepsilon$ runs over $\left(0, \frac{1}{2}\right)$ for instance, we see that the resulting infimum function $\hat{V}$ equals $V$. Similarly (or by symmetry) it follows that the resulting supremum function $\check{V}$ equals $V$. Thus, in this case, we have $\hat{V}=\check{V}=V$ without a Nash equilibrium being attained. This shows that in the definitions (2.20) and (2.21) one cannot omit the requirement on the function $F$ to be superharmonic in $\left\{V<G_{2}\right\}$ and subharmonic in $\left\{V>G_{1}\right\}$, respectively (the «rope cannot be pulled asymptotically»).

Moreover, taking $X$ to be a standard Brownian motion in $[-1,1]$ that is killed at the time of hitting either -1 or 1 , and setting $G_{1}(x)=x^{2}-1$ and $G_{2}(x)=\left(x^{2}-1\right) / 2$ for $x \in[-1,1]$, it is easily seen that the Nash equilibrium holds with $V(x)=G_{2}(x)$ for all $x \in[-1,1]$. Since $\left\{V<G_{2}\right\}=\varnothing$, we see that any finely continuous function $F$ satisfying $G_{1} \leqslant F \leqslant G_{2}$ is superharmonic in $\left\{V<G_{2}\right\}$. This shows that in the definition (2.20) one cannot omit the requirement on the function $F$ to be superharmonic in $\left\{F<G_{2}\right\}$. Similarly (or by symmetry) it follows that in the definition (2.21) one cannot omit the requirement on the function $F$ to be subharmonic in $\left\{F>G_{1}\right\}$.

\section{REFERENCES}

1. Baurdoux E. J., Kyprianou A.E. Further calculations for Israeli options. - Stoch. Stoch. Rep., 2004, v. 76, №6, p. 549-569.

2. Baurdoux E. J., Kyprianou A.E. The McKean stochastic game driven by a spectrally negative Lévy process. - Electron. J. Probab., 2008, v. 13, p. 173-197.

3. Baurdoux E.J., Kyprianou A.E. The Shepp-Shiryaev stochastic game driven by a spectrally negative Lévy process. - Теория вероятн. и ее примен., 2008, т. 53, в. 3, c. $588-609$.

4. Bensoussan A., Friedman A. Nonlinear variational inequalities and differential games with stopping times. - J. Funct. Anal., 1974, v. 16, p. 305-352.

5. Bensoussan A., Friedman A. Nonzero-sum stochastic differential games with stopping times and free boundary problems. - Trans. Amer. Math. Soc., 1977, v. 231, № 2, p. $275-327$. 
6. Blumenthal R. M., Getoor R. K. Markov Processes and Potential Theory. New YorkLondon: Academic Press, 1968, 313 p.

7. Boetius $F$. Bounded variation singular stochastic control and Dynkin game. - SIAM J. Control Optim., 2005, v. 44, № 4, p. 1289-1321.

8. Дьъкин Е.Б. Оптимальный выбор момента остановки марковского процесса. Докл. АН СССР, 1963, т. 150, № 2, с. 238-240.

9. Дьнкин Е. Б. Марковские процессы. М.: Физматгиз, 1963, 859 с.

10. Дьнкин Е.Б. Игровой вариант задачи об оптимальной остановке. - Докл. АН CCCP, 1969, т. 185, № 1, c. 16-19.

11. Ekström E. Properties of game options. - Math. Methods Oper. Res., 2006, v. 63, № 2, p. 221-238.

12. Ekström E., Peskir G. Optimal stopping games for Markov processes. - SIAM J. Control Optim., 2008, v. 47, № 2, p. 684-702.

13. Ekström E., Villeneuve S. On the value of optimal stopping games. - Ann. Appl. Probab., 2006, v. 16, № 3, p. 1576-1596.

14. Элбакидзе Н. В. Построение цены и оптимальных политик в игровой задаче остановки марковского процесса. - Теория вероятн. и ее примен., 1976, т. 21, в. 1, c. $164-169$.

15. Фрид Е. Б. Оптимальная остановка цепи Маркова двумя лицами с противоположными интересами. - Теория вероятн. и ее примен., 1969, т. 14, в. 4, с. 746-749.

16. Friedman A. Stochastic games and variational inequalities. - Arch. Rational Mech. Anal., 1973, v. 51, p. 321-346.

17. Gapeev P.V. The spread option optimal stopping game. - Exotic Option Pricing and Advanced Lévy Models. Chichester: Wiley, 2005, p. 293-305.

18. Гусейн-заде С. М. Об одной игре, связанной с винеровским процессом. - Теория вероятн. и ее примен., 1969, т. 14, в. 4, с. 732-735.

19. Кифер Ю.И. Оптимальная остановка в играх с непрерывным временем. - Теория вероятн. и ее примен., 1971, т. 16 , в. 3, с. 556-562.

20. Kifer Y. Game options. - Finance Stoch., 2000, v. 4, № 4, p. 443-463.

21. Kühn C., Kyprianou A.E. Callable puts as composite exotic options. - Math. Finance, 2007 , v. 17 , № 4, p. 487-502.

22. Kühn C., Kyprianou A.E., van Schaik K. Pricing Israeli options: a pathwise approach. - Stochastics, 2006, v. 79, № 1-2, p. 117-137.

23. Kyprianou A. E. Some calculations for Israeli options. - Finance Stoch., 2004, v. 8, № 1, p. 73-86.

24. Laraki $R$., Solan $E$. The value of zero-sum stopping games in continuous time. SIAM J. Control Optim., 2005, v. 43, № 5, p. 1913-1922.

25. Lepeltier J.P., Maingueneau M.A. Le jeu de Dynkin en théorie générale sans l'hypothèse de Mokobodski. - Stochastics, 1984, v. 13, № 1-2, p. 25-44.

26. Nagai $H$. Non-zero-sum stopping games of symmetric Markov processes. - Probab. Theory Related Fields, 1987, v. 75, № 4, p. 487-497.

27. Peskir G., Shiryaev A.N. Optimal Stopping and Free-Boundary Problems. Basel: Birkhäuser, 2006, 500 p. (Lectures in Mathematics ETH Zürich.)

28. Ширяев A.H. Статистический последовательный анализ: Оптимальные правила остановки. М.: Наука, 1976, 272 с.

29. Snell J. L. Applications of martingale system theorems. - Trans. Amer. Math. Soc., 1952 , v. 73 , p. $293-312$.

30. Stettner L. Zero-sum Markov games with stopping and impulsive strategies. - Appl. Math. Optim., 1982, v. 9, № 1, p. 1-24.

31. Stettner $E$. On closedness of general zero-sum stopping game. - Bull. Polish Acad. Sci. Math., 1984, v. 32, № 5-6, p. 351-361. 